\title{
On air-entraining flow in pump's sump with a vertical suction pipe below critical submergence
}

\author{
Katsuya HIRATA*, Masakatsu HATTORI *, Akira MORITA*, \\ Tsuyoshi MAEDA* and Takashi NOGUCHI** \\ *Department of Mechanical Engineering, Doshisha University, Kyoto 610-0321, Japan \\ E-mail: khirata@mail.doshisha.ac.jp \\ **Department of Aeronautics and Astronautics, Kyoto University, Kyoto 606-8501, Japan
}

Received: 24 August 2018; Revised: 30 November 2018; Accepted: 12 February 2019

\begin{abstract}
In pump's sumps, the air entrainment sometimes occurs. Such flow accompanying the air entrainment becomes complicated owing to both of its unsteadiness with poor periodicity and its fully-three-dimensionality. In various industrial and environmental problems, the air entrainment often induces vibration, noise, low pumping efficiency or pump's collapse at the worst, and is usually complicated owing to both their unsteadiness with poor periodicity and their fully-three-dimensionality. The present aim is to understand the air entrainment into a suction pipe, which appears inside a simple and basic suction sump in the vertical-wet-pit-pump configuration. In particular, we focus upon the influences of governing parameters upon the occurrence-time ratio $\gamma$ of the air entrainment in over-critical-submergence condition, using a conductance-type electric sensor which can detect the existence of air bubbles through a suction pipe with no disturbances by the sensor probe and with a fine spatial resolution in order to achieve accurate measurements. As a result, we reveal the influences of such three kinetic parameters as the Froude number, the Reynolds number and the Weber number (or the Bond number) together with four geometric parameters upon the air entrainment and the free-surface pattern inside the suction sump.
\end{abstract}

Keywords : Pump, Suction sump, Open sump, Water intake, Three-dimensional flow, Two-phase flow, Unsteady flow, Air entrainment

\section{Introduction}

We deal with the flow into a suction pipe which is vertically inserted down into a suction sump across a mean free-water surface. This configuration is conventionally referred to as the "vertical wet-pit pump" or the "vertical-suspended wet-well pump," and has many practical advantages in construction, maintenance and operation. Most of the flows appearing in various industrial and environmental problems like the present suction-sump flow become often complicated owing to both of their unsteadiness with poor periodicity and their fully-three-dimensionality.

In power generation plants, irrigations, drainages and so on, the optimum designs of suction sumps have been needed to get low initial/running costs, compact scale, high efficiency and high performance. In recent years, we commonly require higher-level solutions to satisfy those needs. In such situations, the air entrainment into suction-pipe intakes becomes easy to occur, which often induces vibration, noise, low pumping efficiency or pump's collapse at the worst. Then, we should carefully design the suction sumps and suction pipes to prevent the air entrainment (Yamada, 1979; Tagomori, 1981; Toyokura et al., 1984; Enomoto et al., 1995; Claxton et al., 1998; Kamemoto et al., 2005; Matsui \& Kawane, 2009; Zhao, 2009).

As the air entrainment inside suction sumps is an important concern in practical aspects, there have been many researches about the air entrainment. Among such past studies, coherent dimensional-analysis approaches only concern 
the simplest geometry such as the "horizontal orifice," owing to the applicability of their systematic approaches to such a simple flow with less geometric parameters. However, actual suction sumps usually have more complicated geometries, due to broad-spectrum restrictions and requirements in practical aspects. And unfortunately, we have not enough knowledge even for the flow in a slightly-complicated-geometry suction sump such as a simple vertical-wet-pit-pump-configuration one. Concerning the vertical-wet-pit-pump configuration, researchers have carried out experimental and numerical studies (Iversen, 1953; Denny, 1956; Tagomori, 1979; Tagomori,1980; Padmanabhan et al., 1984; Tagomori \& Ueda, 1991; Okamoto et al., 1991; Oshima \& Tagomori, 1992; Kuwakino, 1993; Nagahara et al., 2002; Iwano et al., 2002; Ansar, 2002; Li et al., 2004; Tang et al., 2011; Zhao \& Nohmi, 2011; Kanemori \& Pan 2015; Yamasaki et al., 2017) in addition to us (Funaki et al., 2008; Hirata et al., 2009; Hirata et al., 2013; Hirata et al., 2016). These studies suggest that we should know the flow and the air-entrainment mechanism more precisely, to prevent the air entrainment.

Our present purpose is to get accurate measurements of the air entrainment into a suction pipe in the vertical-wet-pit-pump configuration. Until now, we have usually judged the air-entrainment occurrence by means of a visually-based method (hereinafter, referred to as a visual method) (Toyokura et al., 1984; Claxton et al., 1998; Zhao, 2009). This conventional visual method has been used prevailingly, because of its practicability and simplicity. However, because the visual method inherently means just the observation of free surfaces, we do not identify the air entrainment itself (will be described later). In our previous study (Hirata et al., 2009), we developed a new and simple conductance-type electric bubble sensor, which can detect the existence of air bubbles through a suction pipe with no disturbances by sensor probes and with a fine spatial resolution. In the present study, we especially focus upon occurrence-time ratio $\gamma$ of the air entrainment as well as Hirata et al. (2009) using the developed bubble sensor, not in the critical-submergence condition which is examined in Hirata et al. (2009) but in over-critical-submergence condition. In addition, we simultaneously observe free-surface pattern inside the suction sump to discuss bubble-sensor measurements with flow inside the suction sump.

\title{
2. Experimental Method
}

\subsection{Experimental apparatus}

Figure 1 shows the present model, which is a simple system of a suction sump and a suction pipe in the vertical-wet-pit-pump configuration. $D$ and $d$ are the outer and inner diameters of the suction pipe, respectively. A former is used as a characteristic length scale. The latter is fixed to $0.9 D$. The suction-pipe intake has a bell-mouth shape. - According to Tagomori (1979), the influence of the bell-mouth shape upon $S_{\mathrm{c}} / D$ is negligible, when $D / d \leq$ 1.75 , where the critical submergence $S_{\mathrm{c}}$ is defined as the minimum value of $S$ with the air entrainment (see Hirata et al., (2009)). In the present study, $D / d$ is fixed to $1 / 0.9(\simeq 1.1)$. Then, it is expected that the influences of the bell-mouth shape upon air entrainment are negligible even at $S / D<S_{\mathrm{c}} / D$. Those influences upon $S_{\mathrm{c}} / D$ and air entrainment are considered to be closely related with the vortex formed near a bell mouth (see Matsui (2016)). Actually, we cannot clearly observe the vortex formed near the bell mouth in the present study.-

The pipe is placed vertically on the centre line of the suction sump. $B, X$ and $Z$ denote the breadth of the suction sump, the clearance from the suction-pipe centre to the suction-sump back wall and the clearance between the suction-pipe intake and the suction-sump bottom wall, respectively. $H$ is water level, namely, the height of a mean water-free surface, then the pipe's submergence depth $S$ is equal to $(H-Z)$.

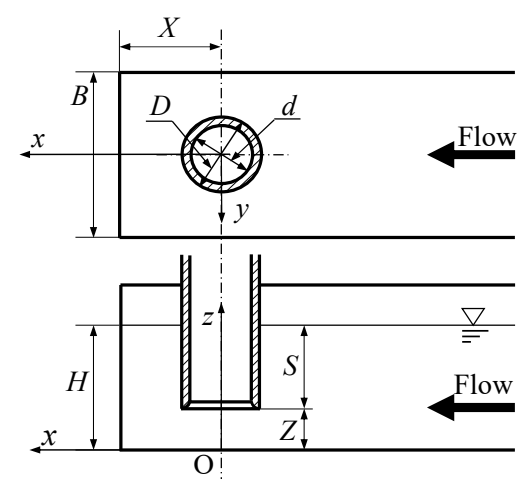

\author{
$D$ : Outside diameter of \\ a suction pipe \\ $d$ : Inside diameter of \\ a suction pipe \\ $B$ : Sump breadth \\ $X$ : Back clearance \\ $H$ : Water level \\ $S$ : Submergence depth \\ $Z$ : Bottom clearance
}

Fig. 1 Model: suction sump and suction pipe in the vertical-wet-pit-pump configuration, together with coordinate system. 
Table 1 Experimental parameters.

\begin{tabular}{c|c}
\hline \hline$D[\mathrm{~mm}]$ & $24-43$ \\
\hline$d[\mathrm{~mm}]$ & $21-38$ \\
\hline$B / D$ & $2.11-5.00$ \\
\hline$X / D$ & $1.43-2.52$ \\
\hline$Z / D$ & $0.26-1.43$ \\
\hline$S / D$ & $0.2-2.5$ \\
\hline$F r$ & $1.2-2.8$ \\
\hline$R e$ & $2.8 \times 10^{4}-5.0 \times 10^{4}$ \\
\hline$\left(R e / F r^{2}\right)$ & $\left(4.1 \times 10^{3}-2.2 \times 10^{4}\right)$ \\
\hline$W e$ & $19-25$ \\
\hline$(B o)$ & $(80-260)$ \\
\hline
\end{tabular}

In order to specify model's dimensions, we define four non-dimensional geometric parameters; that is, a reduced sump breadth $B / D$, a reduced back clearance $X / D$, a reduced bottom clearance $Z / D$ and a reduced submergence depth $S / D$.

The origin of the present coordinate system, the Cartesian coordinates $x, y$ and $z$, is on the axis of the suction pipe and on the suction-sump bottom. The $x$ axis is in the direction of the mainstream inside the suction sump. The $y$ axis is horizontal and perpendicular to the mainstream. And, the $z$ axis is vertical.

In order to specify dynamical state, we define three non-dimensional kinetic parameters; that is the Froude number $F r$, the Reynolds number $R e$ and the Weber number $W e$. They are defined as follows.

$$
\begin{aligned}
& F r=V_{\mathrm{i}} /(g D)^{0.5} \\
& \operatorname{Re}=V_{\mathrm{i}} D / v,
\end{aligned}
$$

and

$$
W e=V_{\mathrm{i}}(\rho D / \sigma)^{0.5},
$$

where $g, v, \rho$ and $\sigma$ denote the gravitational acceralation, kinetic viscosity, fluid density and water-to-air surface tension, respectively. A characteristic velocity scale is the mean flow velocity $V_{\mathrm{i}}$ at the suction-pipe intake which is defined as

$$
V_{\mathrm{i}}=4 Q /\left(\pi D^{2}\right),
$$

where $Q$ is the flow rate into the suction pipe. As supplementary parameters, we use $R e / F r^{2}$ instead of Re and the Bond number such as

$$
B o=\rho g D^{2} / \sigma \text {, }
$$

instead of We. When we define non-dimensional governing parameters, we can consider various options in the choice of characteristic scales. In the present study, we choose $D$ and $V_{\mathrm{i}}$ as length and velocity scales, respectively (see Hirata et al. (2013) for the validity of these choices). In Table 1, we summarise the present values of experimental parameters.

The present experimental apparatus is similar with Hirata et al. (2013). A turbo pump feeds working fluid (water) to a suction sump from a reservoir tank. We control the flow rate from another pump by a valve, and then control the water level inside the suction sump. At the upstream of the suction sump, namely, at $0.84 \mathrm{~m}$ upstream from the back wall of the suction sump, we put a strainer to make flow uniform. The jet pump pumps up water from the suction sump into a suction pipe. We should note that the jet pump has less swirling component, than ordinary turbo pumps. The water from both the suction pipe and the jet pump returns back into the reservoir. We measure the flow rate $Q$ into the suction pipe from the suction sump using a triangle weir or a platform scale. To control surface tension, we add surfactant to water in some experiments (for the details, see Hirata et al. (2013)).

\subsection{Free-surface patterns}

In addition to the bubble-sensor measurements, we simultaneously conduct the observation of the free-surface pattern in a suction sump. Referring to Toyokura et al. (1984), Iversen (1953), Denny (1956), Prosser et al. (1977), 
Rajagopal (1981) and Padmanabhan et al. (1984) together with our preliminary observation, we have classified distinctive free-surface patterns inside the suction sump into four types (a)-(d) (Hirata et al., 2013) as shown in Fig. 2 together with their typical photographs. Then, the present classification is rather different from Claxton et al. (1998) (also see Hirata et al. (2013)). The free-surface pattern inside the suction sump usually shifts from the type (a) to the type (d), as the submergence depth $S$ decreases.

We should note that the above classification is quite conventional, being based on the visual observation of the free surfaces between air and water. Then, each type can be directly connected with neither the occurrence of the air entrainment nor the volume of the entrained air, in a strict sense.

The visual method is the most common method to judge the air-entrainment occurrence. However, due to ambiguous definitions of the air-entrainment occurrence, there has existed some confusion among researchers. (The main reason for the confusion, of course, comes from the lack of direct relations with the air entrainment itself.) Conventionally, the judgment of the air-entrainment occurrence by the visual method are given by the following manner, which is the same as Hirata et al. (2013). When we observe any spatially-continuous air cores from a free surface to a suction-pipe intake, namely, any underwater air bulks accompanied with so-called "the fully-developed entraining vortices" even at a short instant, we recognise the air-entrainment occurrence (see Fig. 2(c)). On the other hand, when we observe some spatially-intermittent air cores, namely, some underwater air bulks accompanied with so-called "the intermittently-developed entraining vortices," we do not recognise the air-entrainment occurrence (see Fig. 2(b)). In a strict sence, the critical submergence $S_{\mathrm{c}}$ is defined as the minimum value of $S$ with the air entrainment. Then, the boundary between the types (b) and (c) should be at $S=S_{\mathrm{c}}$. And, this is confirmed in our previous study (Hirata et al., 2009).

Incidentally speaking, such a judgment manner is more objective and keeps a good reproductivity which has been confirmed in many preparatory experiments, although it lacks a thoroughly-theoretical background. (In fact, we have also confirmed that the results obtained with much longer sampling times than $300 \mathrm{sec}$ almost coincide with the present results.) The present judgment manner is the same as Hirata et al. (2013). Even if we use a somewhat-deferent judgment manner from the present one, the obtained results are considered to be qualitatively identical with the present results (also see Hirata et al. (2013)).

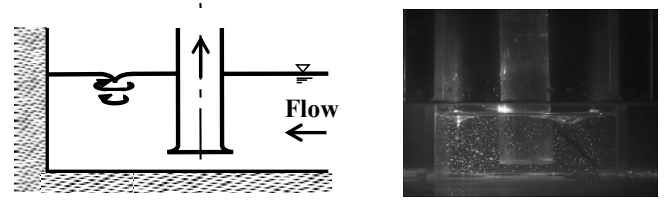

(a) Type (a): free-surface dimple near a suction pipe

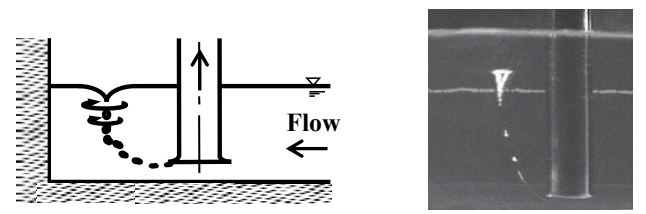

(b) Type (b): suction pipe spatially intermittence air string near

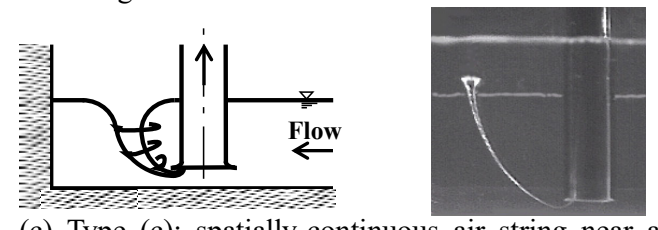

(c) Type (c): spatially-continuous air string near a suction pipe

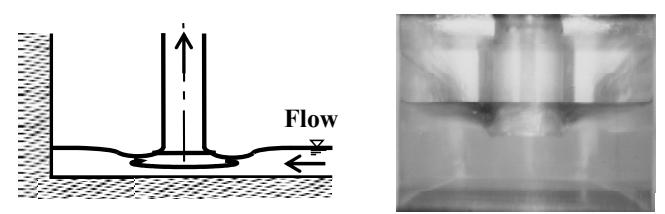

(d) Type (d): free-surface hollow exposed to the peripheral wall of a suction pipe

Fig. 2 Types of distinctive free-surface patterns in suction sump (Hirata et al., 2013), together with photographs.

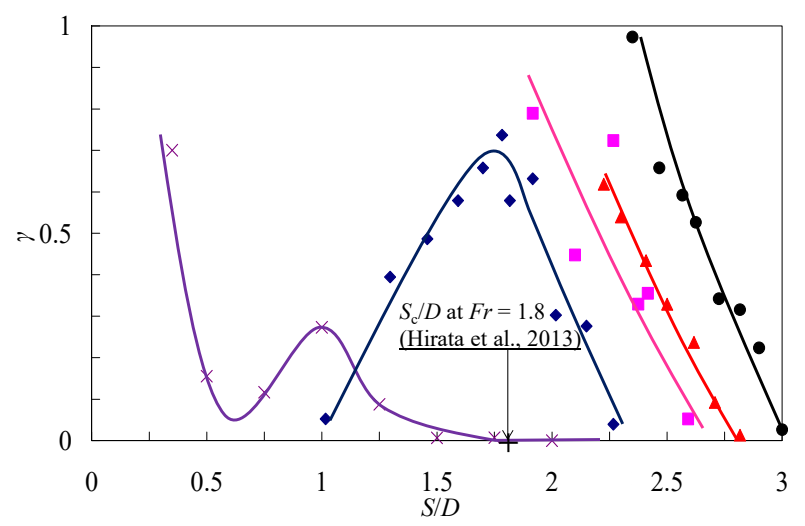

Fig. 3 Air-entrainment occurrence-time ratio $\gamma$ against reduced submergence depth $S / D$.

$$
\begin{gathered}
\times ; B / D=3.15, X / D=1.71, Z / D=0.71, F r=1.8, R e=3.2 \times 10^{4} \\
\left(R e / F r^{2}=9.9 \times 10^{3}\right) \text { and } W e=21(B o=140) . \\
\diamond ; B / D=10, X / D=4, Z / D=3, R e=3.2 \times 10^{4} \text { and } F r=1.0
\end{gathered}
$$

(Okamoto et al., 1991).

; $B / D=10, X / D=4, Z / D=3, \operatorname{Re}=4.8 \times 10^{4}$ and $F r=1.5$

(Okamoto et al., 1991).

$\Delta ; B / D=10, X / D=4, Z / D=3, R e=6.4 \times 10^{4}$ and $F r=1.9$

(Okamoto et al., 1991).

; $B / D=10, X / D=4, Z / D=3, R e=7.2 \times 10^{4}$ and $F r=2.2$

(Okamoto et al., 1991).

$+; S_{\mathrm{C}} / D=1.6 \times F r^{2 / 5}, B / D=3.57, X / D=2.11, Z / D=0.71$, Re

$=3.8 \times 10^{4}>3.0 \times 10^{4}\left(\operatorname{Re}_{F} / r^{2}=1.2 \times 10^{4}>8.0 \times 10^{3}\right)$ and $\mathrm{We}$

$=20-26>12(B o=105-309>95)($ Hirata et al., 2013). 


\subsection{Definition of occurrence-time ratio $\gamma$}

For accurate detection of the air entrainment into the suction pipe, we developed a bubble sensor (for details, see Hirata et al. (2009)). The bubble sensor utilises the same principle as the conductance-type void-ratio meters. By the bubble sensor, we can instantaneously know the occurrence of the air entrainment. More specifically, we choose $1.9 \mathrm{~V}$ as the threshold value of the bubble sensor. By this, we detect the passage of a single air bubble with an air-bubble diameter $\phi>0.15 D(=5.7 \mathrm{~mm})$ as the air entrainment with good accuracy and reproducibility of less-than $10 \%$ error. The error on the output voltage $V$ tends to increase with increasing $\phi$. The increasing error is considered to be related with the air-bubble deformation and the air-bubble eccentricity from the suction-pipe centre with a timely-random fluctuation. The result by the bubble sensor is compared with results by conventional two methods, namely, visual and auditory sensors.

We usually record the output voltage from the bubble sensor for $300 \mathrm{sec}$ in each case. Using this raw time-series data, we calculate an occurrence-time ratio $\gamma$ which represents the ratio of the air-entrainment-occurrence time to the total recording time. Its definition is given by

$\gamma=\frac{\sum_{i=1}^{n} \tau_{i}}{t_{0}}$,

where $t_{0}$ denotes the total recording time which is equal to $300 \mathrm{sec}$. And, $n$ denotes the total number of the air-entrainment occurrence for $t=0-t_{0}$. During a duration time $\tau_{i}$, the $i$-th air entrainment occurs. The air entrainment occurs at any time during the total recording time, when $\gamma=1$. And, the air entrainment never occur, when $\gamma=0$. Supplementarily speaking, one main reason why we consider $\gamma$ is that the phenomenon concerned has a very-weak periodicity, and that $\gamma$ tends to have a constant value for enough large $t_{0}$.

\section{Results and Discussion}

\subsection{Influence of reduced submergence depth $S / D$ upon $\gamma$}

As mentioned in 2.2, we now consider seven governing parameters such as $B / D, X / D, Z / D, S / D, F r, \operatorname{Re}$ and $W e$. Among the seven parameters, we regard $S / D$ as a primary parameter as well as Iversen (1953) and Denny (1956). Actually, the influence of $S / D$ upon $\gamma$ is qualitatively common in many cases, and the corresponding free-surface pattern is as well in many cases. (This will be shown later.) Then, we hereinafter investigate the influence of $S / D$ upon $\gamma$ at first. Next, we investigate the influences of the other parameters upon the relation between $S / D$ and $\gamma$.

Figure 3 shows a typical example of the relation between $S / D$ and $\gamma$, namely, the occurrence-time ratio $\gamma$ plotted against the reduced submergence depth $S / D$, together with another research (Okamoto et al., 1991). In addition, the figure also shows the critical value $S_{\mathrm{c}} / D$ of the reduced submergence depth obtained in our previous study (Hirata et al., 2013).

As $S / D$ decreases from 2.0 to $1.0, \gamma$ monotonically increases. On the other hand, as $S / D$ decreases from 1.0 to $0.7, \gamma$ monotonically decreases. Thus, $\gamma$ attains the maximum at $S / D \simeq 1.0$. From a physical point of view, we can straightforwardly understand the increasing $\gamma$ with decreasing $S / D$ at $S / D=1.0-2.0$, once we remind that the difference of potential energy between the suction-pipe intake and the mean suction-sump free surface is in proportion to $S / D$. Then, the air entrainment becomes easy to occur, as $S / D$ decreases. On the other hand, too small $S / D$ tends to induce three-dimensional flow, breaking two-dimensionality of flow. If the flow in the types (a) - (c) is mainly featured by two-dimensional flow, the air entrainment is prevented at too small $S / D$. In this context, the decreasing $\gamma$ with decreasing $S / D$ at $S / D=0.7-1.0$ is consider to reflect the two-dimensionality breaking. On the balance between the above two, the maximum $\gamma$ is regarded to appear at $S / D \simeq 1.0$.

At $S / D<0.7, \gamma$ progressively increases with decreasing $S / D$. Then, at $S / D \simeq 0.7, \gamma$ attains the minimum which is close to zero. We can also observe shifts of free-surface pattern from the type (a) to the type (d), corresponding to the above behaviour of $\gamma$ with decreasing $S / D$. Specifically speaking, the type (a) or (b) appears in a range of $S / D>1.8$. The type (c) appears in a range of $S / D=0.7-1.8$. Then, the critical submergence depth $S_{\mathrm{c}} / D$ in reduced form becomes about 1.8, which consides with (Hirata et al., 2013). At $S / D<0.7$, the type (d) appears, instead of the type (c). Then at $S / D \simeq 0.7$, there exists a free-surface-pattern shift between the types (c) and (d). Both the above relation between $S / D$ and $\gamma$ and the above relation between $S / D$ and free-surface pattern become common bases, when we will discuss the influences of the other parameters than $S / D$ in the following subsection. 


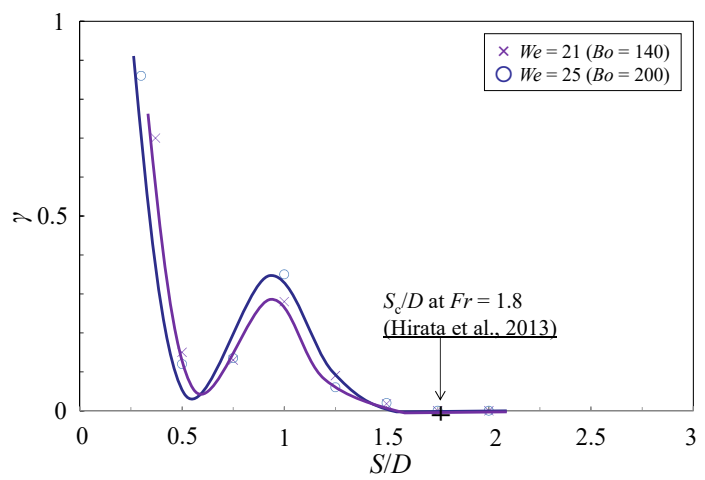

(a) At $F r=1.8$.

$\times ; B / D=3.15, X / D=1.71, Z / D=0.71, F r=1.8, R e=3.2 \times 10^{4}\left(R e / F r^{2}=9.9 \times 10^{3}\right)$ and $W e=21(B o=140)$.

$\bigcirc ; B / D=3.15, X / D=1.71, Z / D=0.71, F r=1.8, R e=3.2 \times 10^{4}\left(R e / F r^{2}=9.9 \times 10^{3}\right)$ and $W e=25(B o=200)$.

$+; S_{\mathrm{c}} / D=1.6 \times F r^{2 / 5}, B / D=3.57, X / D=2.11, Z / D=0.71, R e=3.8 \times 10^{4}>3.0 \times 10^{4}\left(R e / F r^{2}=1.2 \times 10^{4}>8.0 \times 10^{3}\right)$ and $W e$

$=20-26>12(B o=105-309>95)($ Hirata et al., 2013).

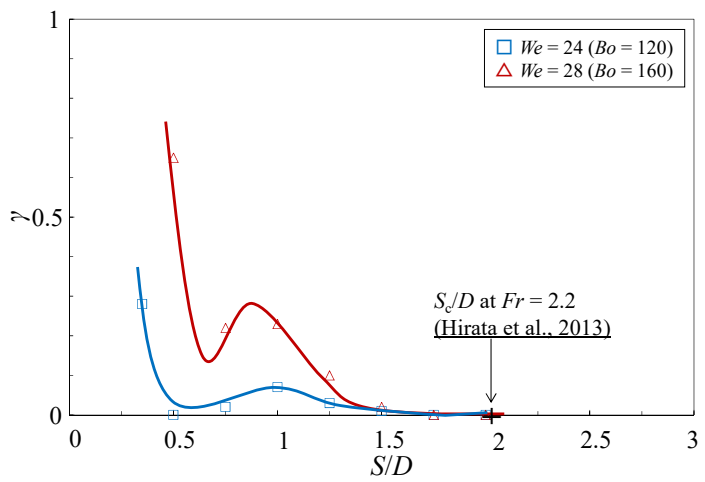

(b) At $F r=2.2$

$\square ; B / D=3.15, X / D=1.71, Z / D=0.71, F r=2.2, R e=3.2 \times 10^{4}\left(R e / F r^{2}=6.6 \times 10^{3}\right)$ and $W e=24(B o=120)$.

$\triangle ; B / D=3.15, X / D=1.71, Z / D=0.71, F r=2.2, R e=3.2 \times 10^{4}\left(R e / F r^{2}=6.6 \times 10^{3}\right)$ and $W e=28(B o=160)$.

$+; S_{\mathrm{c}} / D=1.6 \times F r^{2 / 5}, B / D=3.57, X / D=2.11, Z / D=0.71, R e=3.8 \times 10^{4}>3.0 \times 10^{4}\left(\operatorname{Re}_{F} / F r^{2}=7.9 \times 10^{3}>8.0 \times 10^{3}\right)$ and

$W e=20-26>12(B o=105-309>95)($ Hirata et al., 2013).

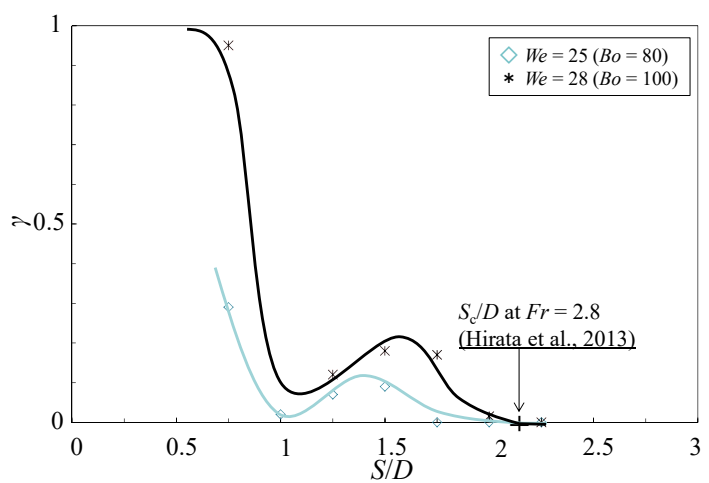

(c) At $F r=2.8$

$\checkmark ; B / D=3.15, X / D=1.71, Z / D=0.71, F r=2.8, R e=3.2 \times 10^{4}\left(\operatorname{Re}_{F} F r^{2}=4.1 \times 10^{3}\right)$ and $W e=25(B o=80)$.

$* ; B / D=3.15, X / D=1.71, Z / D=0.71, F r=2.8, R e=3.2 \times 10^{4}\left(R e / F r^{2}=4.1 \times 10^{3}\right)$ and $W e=28(B o=100)$.

$+; S_{\mathrm{c}} / D=1.6 \times F r^{2 / 5}, B / D=3.57, X / D=2.11, Z / D=0.71, R e=3.8 \times 10^{4}>3.0 \times 10^{4}\left(\operatorname{Re}_{F} / F r^{2}=4.8 \times 10^{3}>8.0 \times 10^{3}\right)$ and

$W e=20-26>12(B o=105-309>95)($ Hirata et al., 2013).

Fig. 4 We effect (or Bo effect) on air-entrainment occurrence-time ratio $\gamma$. 
Both the above relations are qualitatively the identical as Iversen (1953) and Denny (1956) and as Okamoto et al. (1991) at $S / D>1.0$. From a quantitative point of view, the discrepancies of $\gamma$ between the present study and previous ones could be due to different governing parameters in addition to different measuring methods of $\gamma$.

\subsection{Influences of the other governing parameters}

In this subsection, we discuss the influences of the other parameters than $S / D$, on the basis of the relation between $S / D$ and $\gamma$ together with the relation between $S / D$ and free-surface pattern. At first, we will reveal the influences of three kinetic parameters $F r, R e$ and $W e$. Then, we will reveal those of three geometric parameters $B / D, X / D$ and $Z / D$. In the former, we fix the values of the three geometric parameters such as $B / D=3.15, X / D=1.71$ and $Z / D=0.71$, where the air entrainment is easy to occur. In the latter, we fix the values of the three kinetic parameters such as $F r=1.8, R e=$ $3.2 \times 10^{4}\left(\operatorname{Re}_{F} \mathrm{Fr}^{2}=9.9 \times 10^{3}\right)$ and $W e=21(B o=140)$, where we expect that the influences of $\operatorname{Re}\left(\right.$ or $\left.\operatorname{Re} / F r^{2}\right)$ and $W e($ or $B o$ ) are negligible according to our previous study (Hirata et al., 2013) on the critical submergence.

\subsubsection{Influence of Weber number We (or Bond number Bo)}

We now fix the values of $B / D, X / D, Z / D, F r$ and $R e$, and compare the results at different two values of $W e$ (or $B o$ ). Figure 4 shows $W e$ effect (or $B o$ effect) upon $\gamma$, namely, $\gamma$ plotted against $S / D$, together with the $S_{\mathrm{c}} / D$ obtained in our previous study (Hirata et al., 2013). Figures (a), (b) and (c) represent the results at $F r=1.8,2.2$ and 2.8, respectively.

At first, we see Fig. 4(a). The result at $W e=21(B o=140)$ is the same as that in Fig. 3. The result at $W e=25(B o=$ 200) almost coincides with that at $W e=21$, from both qualitative and quantitative viewpoints. Furthermore, we can confirm that the corresponding free-surface patterns at $W e=25$ are the same as those at $W e=21$. As a result, the influences of $W e$ (or $B o$ ) upon $\gamma$ and upon flow pattern are negligible at $W e>21$ (Bo $>140$ ) even at $S / D<S_{\mathrm{c}} / D$. This result is consistent with our previous study (Hirata et al., 2013) where $S_{\mathrm{c}} / D$ is independent of both $W e$ and $R e$ at $W e>$ $12(B o>95)$ and $\operatorname{Re}>3.0 \times 10^{4}\left(\operatorname{Re}_{F} / F^{2}>8.0 \times 10^{3}\right)$.

Second, we see Fig. 4(b). The result at $W e=24(B o=120)$ is similar with that at $W e=28(B o=160)$. In addition, the corresponding free-surface patterns at $W e=24$ are the same as those at $W e=28$. However from a quantitative point of view, we can see a clear difference between both the results. In other words, with decreasing $W e, \gamma$ tends to decrease, then the air entrainment tends to be prevented. This tendency is acceptable, when we remind that surface tension becomes strong with decreasing $W e$ (or $B o$ ). Besides, we can see another clear difference. That is, the values of $S / D$ where $\gamma$ attains the maximum/minimum depends upon $W e$ (or $B o$ ).

Finally, we see Fig. 4(c). Again, the result at $W e=25(B o=80)$ is similar with that at $W e=28(B o=100)$. In addition, the free-surface patterns at $W e=25$ are the same as those at $W e=28$. However from a quantitative point of view, we can see the two clear differences between both the results, as well as Fig. 4(b).

In summary, we can ignore the influences of $W e$ (or $B o$ ) $\gamma$ and free-surface pattern at $F r \lesssim 2$ and $W e>21$ (Bo $>$ 140 ) even at $S / D<S_{\mathrm{c}} / D$. On the other hand, at $F r \gtrsim 2$, we cannot find out the lower limit of $W e$ (or $B o$ ) over which we can ignore the influences of $W e$ (or $B o$ ) upon $\gamma$ and free-surface pattern. At least, as this is based on experiments in the present parameters' ranges examined, further work is needed and useful for more qualitative and quantitative discussion.

\subsubsection{Influence of Reynolds number $\boldsymbol{R e}$}

We now fix the values of $B / D, X / D, Z / D$ and $F r$, and compare the results at different three values of $\operatorname{Re}\left(\right.$ or $R e / F r^{2}$ ). Figure 5 shows the $R e$ effect (or $\operatorname{Re} / F r^{2}$ effect) upon $\gamma$, namely, $\gamma$ plotted against $S / D$ at three values of $\operatorname{Re}$ (or $R e / F r^{2}$ ), together with the $S_{\mathrm{c}} / D$ obtained in our previous study (Hirata et al., 2013). The result at $\operatorname{Re}=3.2 \times 10^{4}\left(\operatorname{Re} / \mathrm{Fr}^{2}=\right.$ $9.9 \times 10^{3}$ ) is the same as that in Fig. 3. Rigorously speaking, we cannot fix $W e$ (or $B o$ ) to the same value for the results at three different $R e$ 's. However, it is acceptable to discuss the influences of Re by comparing the results at $W e=21$ ( $B o=$ $140)$ and $W e=28(B o=250)$, once we remind such a conclusion in 3.2.1 as we can ignore the influences of $W e$ (or $B o$ ) at $F r \lesssim 2$ and $W e>21(B o>140)$.

All the results at three Re's almost coincide with one another, from a qualitative point of view. Furthermore, we can confirm that the corresponding free-surface patterns at three $R e$ 's are the same. Specifically speaking, the result at $R e=$ $5.0 \times 10^{4}\left(R e / F r^{2}=1.5 \times 10^{4}\right)$ and $\operatorname{Re}=2.8 \times 10^{4}\left(\operatorname{Re}_{F} F r^{2}=8.6 \times 10^{3}\right)$ is similar with that at $\operatorname{Re}=3.2 \times 10^{4}\left(\operatorname{Re}_{F} F r^{2}=9.9 \times 10^{3}\right)$. In addition, the corresponding free-surface patterns at $R e=5.0 \times 10^{4}$ and $R e=2.8 \times 10^{4}$ are as well as that at $R e=$ $3.2 \times 10^{4}$. 


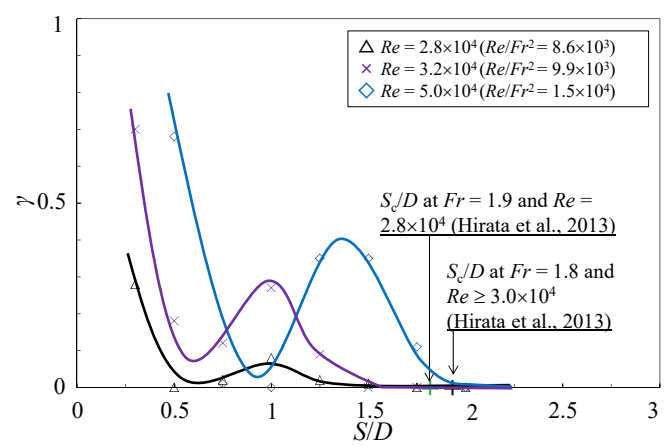

Fig. 5 Re effect on air-entrainment occurrence-time ratio $\gamma$.

$\triangle ; B / D=3.15, X / D=1.71, Z / D=0.71, F r=1.8, R e=2.8 \times 10^{4}\left(R e / F r^{2}=8.6 \times 10^{3}\right)$ and $W e=20(B o=120)$.

$\square ; B / D=3.15, X / D=1.71, Z / D=0.71, F r=1.8, R e=3.2 \times 10^{4}\left(R e / F r^{2}=9.9 \times 10^{3}\right)$ and $W e=21(B o=140)$.

$\checkmark ; B / D=3.15, X / D=1.71, Z / D=0.71, F r=1.8, R e=5.0 \times 10^{4}\left(R e / F r^{2}=1.5 \times 10^{4}\right)$ and $W e=28(B o=250)$.

$+; B / D=3.57, X / D=2.11, Z / D=0.71, F r=1.9, R e=2.8 \times 10^{4}\left(R e / F r^{2}=7.7 \times 10^{3}\right)$ and $W e=8.7-27(B o=20-198)$ (Hirata et al., 2013).

$+; S_{\mathrm{c}} / D=1.6 \times F r^{2 / 5}, B / D=3.57, X / D=2.11, Z / D=0.71, R e=3.8 \times 10^{4}>3.0 \times 10^{4}\left(R e / F r^{2}=1.2 \times 10^{4}-1.5 \times 10^{4}>8.0 \times 10^{3}\right)$ and $W e$ $=20-26>12(B o=105-309>95)($ Hirata et al., 2013).

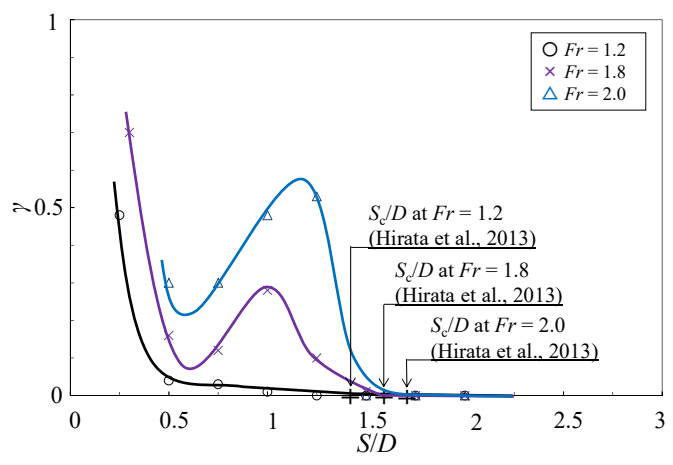

(a) At $F r=1.2-2.0$ and $B o \geq 130$.

$\bigcirc ; B / D=3.15, X / D=1.71, Z / D=0.71, F r=1.2, R e=3.2 \times 10^{4}\left(R e / F r^{2}=2.2 \times 10^{4}\right)$ and $W e=19(B o=260)$.

$\times ; B / D=3.15, X / D=1.71, Z / D=0.71, F r=1.8, R e=3.2 \times 10^{4}\left(\operatorname{Re}_{F} F r^{2}=9.9 \times 10^{3}\right)$ and $W e=21(B o=140)$.

$\triangle ; B / D=3.15, X / D=1.71, Z / D=0.71, F r=2.0, \operatorname{Re}=3.2 \times 10^{4}\left(\operatorname{Re}_{F} F r^{2}=8.0 \times 10^{3}\right)$ and $W e=23(B o=130)$.

$+; S_{\mathrm{c}} / D=1.6 \times F r^{2 / 5}, B / D=3.57, X / D=2.11, Z / D=0.71, R e=3.8 \times 10^{4}>3.0 \times 10^{4}\left(\operatorname{Re}_{F} F r^{2}=9.5 \times 10^{3}-2.6 \times 10^{4}>8.0 \times 10^{3}\right)$ and $W e=$ $20-26>12(B o=105-309>95)$ (Hirata et al., 2013).

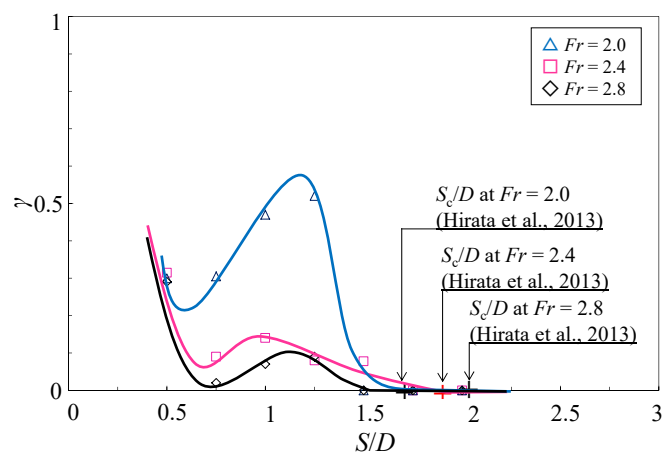

(b) At $F r=2.0-2.8$ and $B o \leq 130$

$\triangle ; B / D=3.15, X / D=1.71, Z / D=0.71, F r=2.0, R e=3.2 \times 10^{4}\left(R e / F r^{2}=8.0 \times 10^{3}\right)$ and $W e=23(B o=130)$.

$\square ; B / D=3.15, X / D=1.71, Z / D=0.71, F r=2.4, R e=3.2 \times 10^{4}\left(R e / F r^{2}=5.6 \times 10^{3}\right)$ and $W e=23(B o=95)$.

$\diamond ; B / D=3.15, X / D=1.71, Z / D=0.71, F r=2.8, R e=3.2 \times 10^{4}\left(R e / F r^{2}=4.1 \times 10^{3}\right)$ and $W e=25(B o=80)$.

$+; S_{\mathrm{c}} / D=1.6 \times F r^{2 / 5}, B / D=3.57, X / D=2.11, Z / D=0.71, R e=3.8 \times 10^{4}>3.0 \times 10^{4}\left(R_{e} / F r^{2}=4.8 \times 10^{3}-9.5 \times 10^{3}>8.0 \times 10^{3}\right)$ and $W e=$ $20-26>12(B o=105-309>95)$ (Hirata et al., 2013).

Fig. $6 \mathrm{Fr}$ effect on air-entrainment occurrence-time ratio $\gamma$. 
However, from a quantitative point of view, we can see a clear difference even between the two results at $R e=$ $3.2 \times 10^{4}\left(R e / F r^{2}=9.9 \times 10^{3}\right)$ and $\operatorname{Re}=5.0 \times 10^{4}\left(\operatorname{Re}_{F} F r^{2}=1.5 \times 10^{4}\right)$ where $F r \lesssim 2$ and $W e>21(B o>140)$, and we cannot ignore the influence of $R e$ (or $R e / F r^{2}$ ). This is contrast to such a conclusion for $S_{\mathrm{c}} / D$ as we can ignore influence of $R e$ (or $R e / F r^{2}$ ) upon $S_{\mathrm{c}} / D$ at $R e>3.0 \times 10^{4}\left(R e / F r^{2}>8.0 \times 10^{3}\right)$. In other words, as $R e$ decreases, $\gamma$ tends to decrease, then the air entrainment tends to be prevented. As well, we see another tendency. As Re decreases, both the values of $S / D$ with the maximum/minimum $\gamma$ 's tend to decrease. These two tendencies are acceptable, once we remind that viscous force comparatively becomes strong with decreasing $R e$.

\subsubsection{Influence of Froude number $\boldsymbol{F r}$}

We now fix the values of $B / D, X / D, Z / D$ and $R e$, and compare the results at different five values of Fr. Figure 6 shows the $F r$ effect upon $\gamma$, namely, $\gamma$ plotted against $S / D$ at five values of $F r$, together with $S_{\mathrm{c}} / D$ obtained in our previous study (Hirata et al., 2013). Figures (a) and (b) represent the results at $F r=1.2-2.0$ and 2.0-2.8, respectively. The result at $F r=1.8$ in Fig. 6(a) is the same as Fig. 3. Rigorously speaking, we cannot fix $W e$ (or $B o$ ) to the same value for the results at five different Fr's, as well as Fig. 5. However, it is acceptable to discuss the influences of $F r$ in Fig. 6(a) at least, once we remind such a conclusion in 3.2.1 as we can ignore the influences of $\mathrm{We}$ (or $\mathrm{Bo}$ ) at $\mathrm{Fr} \lesssim 2$ and $W e>21(B o>140)$ even at $S / D<S_{\mathrm{c}} / D$.

At first, we see Fig. 6(a). The result at $F r=2.0$ is similar with that at $F r=1.8$. In addition, the corresponding free-surface pattern at $F r=2.0$ is as well as that at $F r=1.8$. However, from a quantitative point of view, we can see a clear difference between both the results. On the other hand, the results on $\gamma$ at $F r=1.2$ and the corresponding free-surface pattern are different from those at $F r=1.8$ and 2.0, qualitatively and quantitatively. More specifically, as $S / D$ decreases, $\gamma$ at $F r=1.2$ monotonically increases without the maximum/minimum. About the corresponding free-surface pattern at $F r=1.2$, the type (d) becomes easy to appear in a wide range of $S / D$ at $S / D<S_{\mathrm{c}} / D$, while the type (c) is difficult to be observed at $S / D<S_{\mathrm{c}} / D$. Of course, we cannot ignore the influence of $F \mathrm{r}$ from a quantitative point of view. As a result, we can get a quantitative conclusion on $\gamma$ at $F r=1.2-2.0$ in Fig. 6(a), in spite of the above qualitative discrepancies between the result at $F r=1.2$ and the others. That is to say, as $F r$ decreases, $\gamma$ tends to decrease, then the air entrainment tends to be prevented. This tendency is acceptable, once we remind that gravity force becomes comparatively strong with decreasing Fr. The above quantitative conclusion on $\gamma$ is consistent with such a conclusion on $S_{\mathrm{c}} / D$ as $S_{\mathrm{c}} / D$ monotonically decreases with decreasing $\operatorname{Fr}$ (Hirata et al., 2013) (also see Fig. 6(a)).

Second, we see Fig. 6(b). All the results at three different Fr's ( 2 2.0)are similar with one another. In addition, the corresponding free-surface patterns are the same. However, from a quantitative point of view, we can see clear differences among them. More specifically, as Fr decreases, $\gamma$ tends to increase, then, the air entrainment tends to be prevented. This seems strange, because gravity force becomes comparatively strong with decreasing $F r$. This strangeness might be come from imperfect discussion on pure $F r$ effect. Actually, $F r \gtrsim 2$ in Fig. 6(b).

\subsubsection{Influence of reduced sump breadth $B / D$}

We now fix the values of $X / D, Z / D, F r, \operatorname{Re}$ and $W e$ (or $B o$ ), and compare the results at different four values of $B / D$. Fig. 7 shows $B / D$ effect upon $\gamma$, namely, $\gamma$ plotted against $S / D$ at four values of $B / D$, together with $S_{\mathrm{c}} / D$ obtained in our previous study (Hirata et al., 2013). The result at $B / D=3.15$ is the same as Fig. 3.

From a qualitative point of view, both the behaviour of $\gamma$ with decreasing $S / D$ at $B / D=2.11$ and the corresponding free-surface pattern are the same as those at $F r=1.2$ in Fig. 6 (a). Both at $B / D=4.21$ are the same as those at $B / D=$ 3.15. Again, both at $B / D=5.00$ are the same as those at $F r=1.2$ in Fig. 6(a).

From a quantitative point of view, $\gamma$ at $S / D \simeq 1.0$ is remarkable large at $B / D=3.15$ and 4.21. Corresponding to this, $S_{\mathrm{c}} / D$ 's at $B / D=3.15$ and 4.21 are much larger than those at $B / D=2.11$ and 5.00. The flow pattern at $S / D \simeq 1.0$ is in the type (c) at $B / D=3.15$ and 4.21. And, the flow pattern at $S / D<S_{\mathrm{c}} / D$ is almost in the type (d) at $B / D=2.11$ and 5.00 . In summary, such a condition as $B / D \simeq 3-4$ is suitable for the type (c). Then, $\gamma$ at $B / D \simeq 3-4$ tends to become remarkably large at $S / D \simeq 1.0$, with enhancing the air entrainment.

This condition for the maximum $\gamma$ at $S / D \simeq 1$ is linked to the increase/decrease of $S_{\mathrm{c}} / D$. So, it seems difficult to directly explain such a tendency as $S_{\mathrm{c}} / D$ increases with increasing $B / D$ at $B / D<3.15$. We might consider that this tendency is related to the interference of the suction-sump side walls with the vortex formation in the downstream of the suction pipe (see Funaki et al. (2008)), then would need further researches. On the other hand, it seems possible to understand such a tendency as $S_{\mathrm{c}} / D$ decreases with further increasing $B / D$ at $B / D>4.21$, on the basis of the global $F r$ 


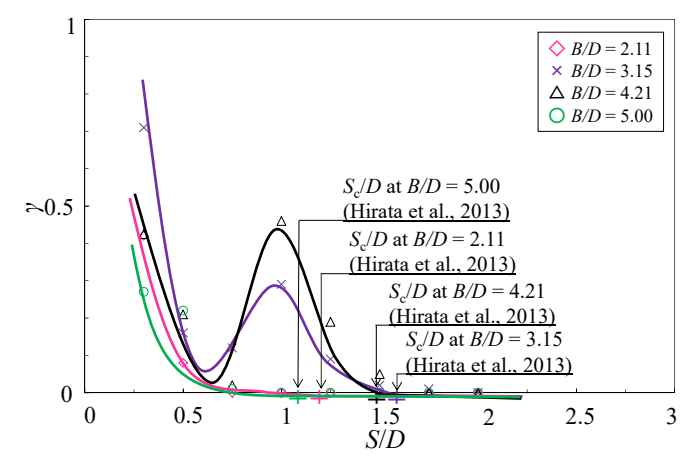

Fig. $7 \mathrm{~B} / \mathrm{D}$ effect on air-entrainment occurrence-time ratio $\gamma$.

$\diamond ; B / D=2.11, X / D=1.71, Z / D=0.71, F r=1.8, R e=3.2 \times 10^{4}\left(R e / F r^{2}=9.9 \times 10^{3}\right)$ and $W e=21(B o=140)$. $\times ; B / D=3.15, X / D=1.71, Z / D=0.71, F r=1.8, R e=3.2 \times 10^{4}\left(\operatorname{Re}_{F r} F^{2}=9.9 \times 10^{3}\right)$ and $W e=21(B o=140)$. $\triangle ; B / D=4.21, X / D=1.71, Z / D=0.71, F r=1.8, R e=3.2 \times 10^{4}\left(R e / F r^{2}=9.9 \times 10^{3}\right)$ and $W e=21(B o=140)$. $\circ ; B / D=5.00, X / D=1.71, Z / D=0.71, F r=1.8, R e=3.2 \times 10^{4}\left(R e / F r^{2}=9.9 \times 10^{3}\right)$ and $W e=21(B o=140)$. $+; B / D=2.11, X / D=1.32, Z / D=0.71, F r=1.6, R e=3.8 \times 10^{4}\left(R e / F r^{2}=1.5 \times 10^{4}\right)$ and $W e=22.4(B o=197)$ (Hirata et al., 2013). $+; B / D=3.15, X / D=1.32, Z / D=0.71, F r=1.6, R e=3.8 \times 10^{4}\left(R e / F r^{2}=1.5 \times 10^{4}\right)$ and $W e=22.4(B o=197)$ (Hirata et al., 2013). $+; B / D=4.21, X / D=1.32, Z / D=0.71, F r=1.6, R e=3.8 \times 10^{4}\left(R e / F r^{2}=1.5 \times 10^{4}\right)$ and $W e=22.4(B o=197)$ (Hirata et al., 2013). $+; B / D=5.00, X / D=1.32, Z / D=0.71, F r=1.6, R e=3.8 \times 10^{4}\left(R e / F r^{2}=1.5 \times 10^{4}\right)$ and $W e=22.4(B o=197)$ (Hirata et al., 2013).

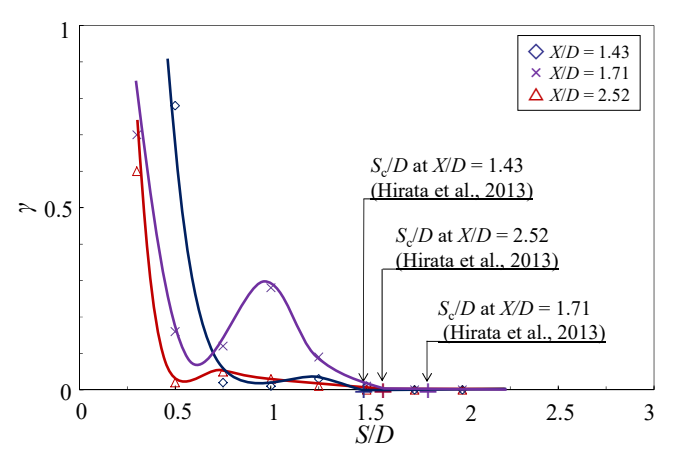

Fig. $8 X / D$ effect on air-entrainment occurrence-time ratio $\gamma$.

$\checkmark ; B / D=3.15, X / D=1.43, Z / D=0.71, F r=1.8, R e=3.2 \times 10^{4}\left(R e / F r^{2}=9.9 \times 10^{3}\right)$ and $W e=21(B o=140)$. $\times ; B / D=3.15, X / D=1.71, Z / D=0.71, F r=1.8, R e=3.2 \times 10^{4}\left(R e / F r^{2}=9.9 \times 10^{3}\right)$ and $W e=21(B o=140)$. $\triangle ; B / D=3.15, X / D=2.52, Z / D=0.71, F r=1.8, \operatorname{Re}=3.2 \times 10^{4}\left(\operatorname{Re}_{F} / F^{2}=9.9 \times 10^{3}\right)$ and $W e=21(B o=140)$. $+; B / D=3.16, X / D=1.43, Z / D=0.71, F r=1.6, R e=3.8 \times 10^{4}\left(R e / F r^{2}=1.5 \times 10^{4}\right)$ and $W e=22.4(B o=197)$ (Hirata et al., 2013). $+; B / D=3.16, X / D=1.71, Z / D=0.71, F r=1.6, R e=3.8 \times 10^{4}\left(R e / F r^{2}=1.5 \times 10^{4}\right)$ and $W e=22.4(B o=197)$ (Hirata et al., 2013). $+; B / D=3.16, X / D=2.52, Z / D=0.71, F r=1.6, R e=3.8 \times 10^{4}\left(R e / F r^{2}=1.5 \times 10^{4}\right)$ and $W e=22.4(B o=197)$ (Hirata et al., 2013).

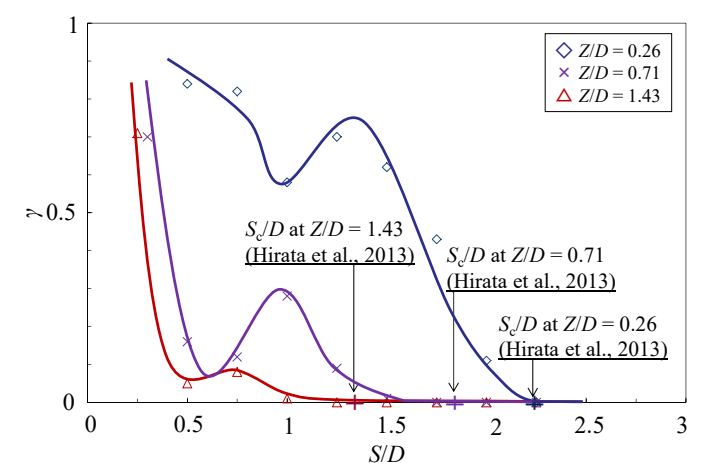

Fig. $9 \mathrm{Z} / \mathrm{D}$ effect on air-entrainment occurrence-time ratio $\gamma$.

$\diamond ; B / D=3.15, X / D=1.71, Z / D=0.26, F r=1.8, R e=3.2 \times 10^{4}\left(R e / F r^{2}=9.9 \times 10^{3}\right)$ and $W e=21(B o=140)$. $\times ; B / D=3.15, X / D=1.71, Z / D=0.71, F r=1.8, R e=3.2 \times 10^{4}\left(\operatorname{Re}_{F} F r^{2}=9.9 \times 10^{3}\right)$ and $W e=21(B o=140)$. $\triangle ; B / D=3.15, X / D=1.71, Z / D=1.43, F r=1.8, R e=3.2 \times 10^{4}\left(R e / F r^{2}=9.9 \times 10^{3}\right)$ and $W e=21(B o=140)$. $+; B / D=3.16, X / D=2.11, Z / D=0.26, F r=1.6, R e=3.8 \times 10^{4}\left(R e / F r^{2}=1.5 \times 10^{4}\right)$ and $W e=22.4(B o=197)$ (Hirata et al., 2013). $+; B / D=3.16, X / D=2.11, Z / D=0.71, F r=1.6, R e=3.8 \times 10^{4}\left(R e / F r^{2}=1.5 \times 10^{4}\right)$ and $W e=22.4(B o=197)$ (Hirata et al., 2013). $+; B / D=3.16, X / D=2.11, Z / D=1.43, F r=1.6, R e=3.8 \times 10^{4}\left(R e / F r^{2}=1.5 \times 10^{4}\right)$ and $W e=22.4(B o=197)$ (Hirata et al., 2013). 
effect upon $S_{\mathrm{c}} / D$. That is to say, local velocities near the suction-pipe intake tend to decrease as $B / D$ increases. This causes the decreases of the corresponding local Froude numbers. If we assume that the effects of such local Froude numbers are similar with the global one, the tendency seems acceptable. Because, the local Froude numbers are considered to be more definitive and effective than the global one on the air entrainment.

\subsubsection{Influence of reduced back clearance $X / D$}

We now fix the values of $B / D, Z / D, F r, \operatorname{Re}$ and $W e$ (or $B o$ ), and compare the results at different three values of $X / D$. Fig. 8 shows $X / D$ effect upon $\gamma$, namely, $\gamma$ plotted against $S / D$ at three values of $X / D$, together with $S_{\mathrm{c}} / D$ obtained in our previous study (Hirata et al., 2013). The result at $X / D=1.71$ is the same as Fig. 3.

From a qualitative point of view, both the behaviour of $\gamma$ with decreasing $S / D$ at $X / D=1.43$ and 2.52 , and the corresponding free-surface patterns are the same as those at $F r=1.2 \mathrm{in} \mathrm{Fig.} \mathrm{6(a).} \mathrm{Then,} \mathrm{the} \mathrm{flow} \mathrm{patterns} \mathrm{at} S / D<S_{\mathcal{C}} D$ are almost in the type (d). As mentioned above, the flow pattern at $X / D=1.71$ is in the type (c) at $S / D \simeq 1.0$, where $\gamma$ attains the maximum.

In summary, such a condition as $X / D \simeq 1.7$ is suitable for the type (c), as well as such a condition as $B / D \simeq 3-4$. Then, $\gamma$ at $X / D \simeq 1.7$ tends to become remarkably large at $S / D \simeq 1.0$, with enhancing the air entrainment. This condition for the maximum $\gamma$ at $S / D \simeq 1.0$ is also linked to the increase/decrease of $S_{\mathrm{c}} / D$. As well as such a tendency as $S_{\mathrm{c}} / D$ increases with increasing $B / D$ from zero to a certain value $(\simeq 3-4)$ and such a tendency as $S_{\mathrm{c}} / D$ decreases with further increasing $B / D$ from the certain value $(\simeq 3-4)$, it seems difficult to directly explain this complicated manner of the $X / D$ effect. To understand this complicated manner, we also need further work concerning the interference/augmentation of the vortex formation in the downstream of the suction pipe with/by the suction-sump back wall (for time-averaged flow in the downstream, see Funaki et al. (2008)).

\subsubsection{Influence of reduced bottom clearance $\boldsymbol{Z} / \boldsymbol{D}$}

We now fix the values of $X / D, B / D, F r, \operatorname{Re}$ and $W e$ (or $B o$ ), and compare the results at different three values of $Z / D$. Fig. 9 shows $Z / D$ effect upon $\gamma$, namely, $\gamma$ plotted against $S / D$ at three values of $Z / D$, together with $S_{\mathrm{c}} / D$ obtained in our previous study (Hirata et al., 2013). The result at $Z / D=0.71$ is the same as Fig. 3.

The results at $Z / D=0.26$ and 1.43 are similar with that at $Z / D=0.71$. In addition, the corresponding free-surface patterns at $Z / D=0.26$ and 1.43 are the same as that at $Z / D=0.71$. However, from a quantitative point of view, we can see clear differences among the three results at $Z / D=0.26, Z / D=0.71$ and $Z / D=1.43$, and we cannot ignore the influence of $Z / D$. In other words, as $Z / D$ increases, $\gamma$ tends to decreases and then the air entrainment tends to be prevented. This tendency is linked to the decrease of both the values of $S / D$ with the maximum/minimum $\gamma$ s, and to the decrease of $S_{\mathrm{c}} / D$. This tendency is acceptable, once we remind that an actual Froude number based on local velocity near the suction-pipe intake cloud become large with decreasing $Z / D$. Because, the local velocity near the suction-pipe intake could be enhanced, as $Z / D$ decreases.

\section{Summary}

In order to understand the air entrainment appearing inside a simple and basic suction sump in the vertical-wet-pit-pump configuration, we have focused on the influences of governing parameters upon the occurrence-time ratio $\gamma$ of the air entrainment, using a conductance-type electric sensor which can detect the existence of air bubbles through a suction pipe with no disturbances by the sensor probe and with a fine spatial resolution in order to achieve accurate measurements.

As a result, we have revealed the influences of three kinetic parameters like the Froude number $F r$, the Reynolds number $R e$ and the Weber number $W e$ (or the Bond number $B o$ ) and three geometric parameters like a reduced sump breadth $B / D$, a reduced back clearance $X / D$ and a reduced bottom clearance $Z / D$ upon the relation between one geometric parameter a reduced submergence depth $S / D$ and the air entrainment in such an over-critical-submergence condition as $S / D<S_{\mathrm{c}} / D$. About the influences of $F r$, both $\gamma$ and free-surface pattern at $F r \lesssim 1$ are different from that at $F r \gtrsim 1$ : namely, the type (c) is difficult to be observed at $F r \lesssim 1$. Of course, we cannot ignore the influences of $F r$ from a quantitative point of view at any time. About the influences of $R e$ and $W e$ (or $B o$ ), we cannot ignore them even at larger values of $R e$ and $W e(B o)$ than those for $S_{\mathrm{c}} / D$. On the other hand, the influences of $B / D, X / D$ and $Z / D$ are well linked to $S_{\mathrm{c}} / D$. About the influences of $B / D$ and $X / D$, both $\gamma$ and the maximum $\gamma$ at $S / D<S_{\mathrm{c}} / D$ corresponds to the suitable condition for the type (c). The influences of $Z / D$ can be explained by the introduction of a local Froude number, 
as well as $S_{\mathrm{c}} / D$.

In the present fundamental approach, we have revealed the influences of governing parameters upon $\gamma$ at $S / D<$ $S_{\mathrm{c}} / D$. Such conclusions are effective and useful to prevent the occurrence of the air entrainment, as the air entrainment tends to be prevented with decreasing $\gamma$. In practical applications with complicated geometry and more number of governing parameters, further researches are desired in future.

\section{Reference}

Answar, M., Nakato, T. and Constantinescu, G., Numerical simulations of inviscid three-dimensional flows at singleand dual- pump intakes, Journal of Hydraulic Research, Vol. 4, No. 4 (2002), pp. 461-470.

Claxton, J. et al., American National Standard for Pump Intake Design (1988 HI Standard, ANSI/HI9.8-1998), Hydraulic Institute, Parsippany, New Jersey, USA (1998).

Denny, D. F., An experimental study of air-entraining vortices in pump sumps, Proceedings of IMechE. Vol. 170 , No. 2 (1956), pp. 106-116.

Enomoto, T., Tagomori, M. and Goto, A., Utilization of CFD in pump design, Turbomachinery, Vol. 23, No. 3 (1995), pp. 165-168 (in Japanese).

Funaki, J., Neya, M., Hattori, M., Tanigawa, H. and Hirata, K., Flow measurements in a suction sump by UVP, Journal of Fluid Science and Technology, JSME, Vol. 3 , No. 1 (2008), pp. 68-79.

Hirata, K., Saitoh, K., Hattori, M., Nakatani, Y. and Funaki, J., Occurrence-time-ratio measurements on air entrainment in a suction sump, Journal of Fluid Science and Technology, JSME, Vol. 4, No. 1 (2009), pp. 47-61.

Hirata, K., Funaki, J., Kubota, Y., Inagaki, K., Taoka, M. and Saida, T, Occurrence condition of air entrainment into a vertical wet-pit pump, Journal of Fluid Science and Technology, JSME, Vol. 8, No. 3 (2013), pp. 364-379.

Hirata, K., Shintani, A., Kawaguchi, R., Inagaki, K., Nagura, T. and Maeda, T., 3D-PTV measurement verified by UVP for unsteady and three-dimensional flow inside a suction sump, Journal of Fluid Science and Technology, JSME, Vol. 11, No. 3 (2016), pp. 1-13.

Iversen, H. W., Studies of submergence requirement of high specific speed pump, Transactons of ASME, Vol. 75, No. 4 (1953), pp. 635-641.

Iwano, R., Shibata, T., Nagahara, T. and Okamura, T., Development of a numerical method for predicting submerged vortex cavitation in a pump sump (Application of the method to steady state flow analysis), Transanctions of JSME (Series B), Vol. 68, No. 667 (2002), pp. 652-657 (in Japanese).

Kamemoto, K. et al., Method for Model Testing the Performance of a Pump Sump (TSJ Standard TSJ S 002), Turbomachinery Society of Japan, Tokyo (2005) (in Japanese).

Kanemori, Y. and Pan, Y., Surface vortex prediction and preventing technique in pump intake sump, Turbomachinery, Vol. 43, No.2 (2015) pp. 90-98 (in Japanese).

Kuwakino, S., On the prevention against air-entraining vortices in a suction sump, Turbomachinery, Vol. 21 , No.6 (1993) pp. 343-349 (in Japanese).

Li, S., Lai, Y., Weber, L., Silva, M. and Patel, C., Validation of a three-dimensional numerical model for water-pump intakes, Journal of Hydraulic Research, Vol. 42, No. 3 pp. 282-292 (2004).

Matsui, J. and Kawane, H., Outline on pump sump and its model test, Turbomachinery Vol. 37, No.1 (2009) pp. 4-11 (in Japanese).

Matsui, J., Similarity on occurrence condition of vortexes in pump sumps, Turbomachinery, Vol. 44, No.4 (2016) pp. 223-230 (in Japanese).

Nagahara, T., Sato, T., Kawabata, S. and Okamura, T., Effect of the submerged vortex cavitation in pump suction intake on mixed flow pump impeller, Turbomachinery, Vol. 30, No.2 (2002) pp. 70-75 (in Japanese).

Okamoto, H., Kamemoto, K. and Nakaguchi, I., Intermittence of the air entraining vortex and the submerged vortex in a suction sump, Transanctions of JSME (Series B), Vol. 57, No. 536 (1991) pp. 1210-1213 (in Japanese).

Oshima, M. and Tagomori, M., On critical dimensions for air-entraining vortices of a suction sump, Turbomachinery Vol. 20, No.4 (1992) pp. 205-210 (in Japanese).

Padmanabhan, M. and Hecker, G. E., Scale effects in pump sump models, Journal of Hydraulic Engineering, ASCE, Vol. 110, No. 11 (1984), pp. 1540-1556.

Prosser, M. J., The Hydraulic Design of Pump Sumps and Intakes, British Hydromechanics Research Association, 
Cranfield, Bedford, UK (1977).

Rajagopal, H. Y., Prevention of vortices at pump intakes, Power Engineering, January (1981) pp. 52-55.

Tagomori, M., Flow pattern and air-entraining vortices in a suction sump, Turbomachinery, Vol. 7, No. 8 (1979), pp. 451-460 (in Japanese).

Tagomori, M., Flow pattern and air-entraining vortices in a suction sump, Turbomachinery, Vol. 8, No. 10 (1980), pp. 579-587 (in Japanese).

Tagomori, M., Factors and prevision for air-entrainment-vortex occurrence, Turbomachinery, Vol. 9, No. 8 (1981), pp. 484-491 (in Japanese).

Tagomori, M. and Ueda, H., An experimental study on submerged vortices and flow pattern in the pump sump, Transanctions of JSME (Series B), Vol. 57, No. 543 (1991), pp. 3641-3646 (in Japanese).

Tang, X. Y., Wang, F. J., Li, Y. J., Cong, G. H., Shi, X. Y., Wu, Y. L. and Qi, L. Y., Numerical investigations of vortex flows and vortex suppression schemes in a large pumping-station sump, Proceedings of IMechE Part C: Journal of Mechanical Engineering Science, Vol. 225, No. 6 (2011), pp. 1459-1480.

Toyokura, T. et al., Standard Method for Model Testing the Performance of a Pump Sump (JSME Standard JSME S 004), JSME, Tokyo (1984) (in Japanese).

Yamada, T., Under-water phenomenon in a pump sump, Turbomachinery, Vol. 7, No. 4 (1979), pp. 241-248 (in Japanese).

Yamasaki, R., Kouchi, T., Nagata, Y. and Yanase, S., OpenFOAM analysis of air-entraining and submerged vortices in pump sump, Transactions of JSME, Vol. 83, No. 853 (2017), pp. 1-13 (in Japanese).

Zhao, L., Visualization of vortices in pump sump, Journal of Visualization Society of Japan Vol. 30, No. 116 (2009) pp. 28-33 (in Japanese).

Zhao, L. and Nohmi, M., Numerical prediction of air-entrained vortex in pump sump using VOF method, Turbomachinery, Vol. 39, No.7 (2011) pp. 425-430 (in Japanese). 